\title{
Scheme for stabilization of output power of an x-ray self-amplified spontaneous emission free-electron laser
}

\author{
Gianluca Geloni, Evgeni Saldin, Evgeni Schneidmiller, and Mikhail Yurkov \\ Deutsches Elektronen-Synchrotron (DESY), Notkestrasse 85, D-22607 Hamburg, Germany
}

(Received 18 September 2008; published 4 December 2008)

\begin{abstract}
Output stability of an XFEL setup is naturally linked to the stability of the linac rf system through the bunch compression process. This fact leads to very tight tolerances for $\mathrm{rf}$ amplitudes and phases. We propose a feedback scheme using an optically modulated electron beam where the compression factor is self-stabilized due to the action of longitudinal space charge fields. Our scheme allows loosening rf tolerances by an order of magnitude. Alternatively, for the same variations of rf parameters, stability of the FEL pulse energy could be improved by an order of magnitude.
\end{abstract}

DOI: 10.1103/PhysRevSTAB.11.120701

PACS numbers: 41.60.Cr, 29.20.-c

\section{INTRODUCTION}

Free-electron lasing at wavelengths shorter than the ultraviolet can be achieved with a single-pass, high-gain FEL amplifier. Because of lack of powerful, coherent seeding sources, short-wavelength FEL amplifiers work in the so-called self-amplified spontaneous emission (SASE) mode, where the amplification process starts from shot noise in the electron beam [1-3]. Present acceleration and FEL techniques hold potential for SASE FELs to generate wavelengths as short as $0.1 \mathrm{~nm}$ [4-6].

Pulse-energy stability of the radiation from a shortwavelength FEL might be challenging due to the fact that one deals with an exponential gain of many orders of magnitude. In particular, there exist both intrinsic and extrinsic fluctuations of the FEL pulse energy.

Intrinsic fluctuations are due to the start-up from shot noise. In the exponential gain regime they scale as $\sqrt{l_{\text {coh }} / \sigma_{z}}$, where $l_{\text {coh }}$ is the FEL coherence length and $\sigma_{z}$ is rms bunch length (see [7] for more details). For hard $\mathrm{x}$-ray FELs [4-6], these fluctuations are not so large because of a short coherence length (of the order of $0.1 \mu \mathrm{m}$ ). Moreover, they are reduced when an FEL reaches saturation. In Fig. 1, the 1D version of the code FAST [8] was used to present the intrinsic rms fluctuations of FEL pulse energy versus undulator length (curve 1 on the lower plot) for the SASE1 undulator, operating at $0.1 \mathrm{~nm}$ [4]. Fluctuations are about $7 \%$ in the exponential gain regime before saturation, and about $2 \%$ at saturation.

Extrinsic fluctuations are due to jitter in amplitude and phase of the rf system. In the high-gain linear regime, the radiation power increases along the undulator length as $\exp \left(2 z / L_{g}\right)$, where $L_{g}$ is the field gain length, which depends on beam and undulator parameters. In the case when the beta function in the undulator is optimized for the highest FEL gain and the energy spread effect is a small correction, $L_{g} \propto \epsilon_{n}^{5 / 6} / I^{1 / 2}$ [9], where $I$ is the beam current and $\epsilon_{n}$ is the normalized emittance. While the emittance is supposed to be a stable parameter in beam formation systems for x-ray FELs, fluctuations of the beam current are of major concern due to a large compression factor $C$ in magnetic chicanes. For instance, in the case of the European XFEL [4], the beam current increases from 50 A to $5 \mathrm{kA}$, i.e. $C=100$. Analysis in [10] shows that $\Delta C / C_{0} \propto C_{0} \Delta x$, where $C_{0}$ is the nominal compression factor and $x$ is a fluctuating rf parameter such as amplitude or phase. In other words, the larger the compression factor, the more sensitive it is to variations of the rf parameters. As a result, estimated typical rf jitter tolerances are very tight, of the order of a hundredth of a degree for phases and of $10^{-4}$ for amplitudes. The rms relative fluctuations of the FEL pulse energy $E, \sigma_{E} / E$, are plotted in Fig. 1 for rms relative fluctuations of compression factor $\sigma_{C} / C_{0}=10 \%$ (here and below we consider a flattop distribution of compression factor variations). One can see that extrinsic fluctuations are much stronger than intrinsic ones. In the exponential gain regime, with $L_{g} \propto I^{-1 / 2}$ one can simply derive for small $\sigma_{C}$ (accounting for variations of the pulse width):

$$
\frac{\sigma_{E}}{E} \simeq\left(\frac{z}{L_{g}}-1\right) \frac{\sigma_{C}}{C_{0}}
$$

This dependence is shown in Fig. 1 with dots.

One should distinguish, here, between jitters and slow drifts of rf parameters. On the one hand, slow drifts can be compensated by a beam-based slow feedback, as it is done at FLASH, a precursor of the European XFEL, where coherent diffraction radiation produced by compressed bunches is used to regulate the phase of accelerating module upstream of the bunch compressor. On the other hand, pulse-to-pulse variations (jitters) cannot be compensated in this way. In this paper we propose a scheme that allows one to dramatically reduce the sensitivity of the FEL pulse energy on the rf parameters variation by developing, in practice, a single-bunch feedback.

Such feedback can only be based on collective fields of each individual bunch. An idea to use resistive wakefields 

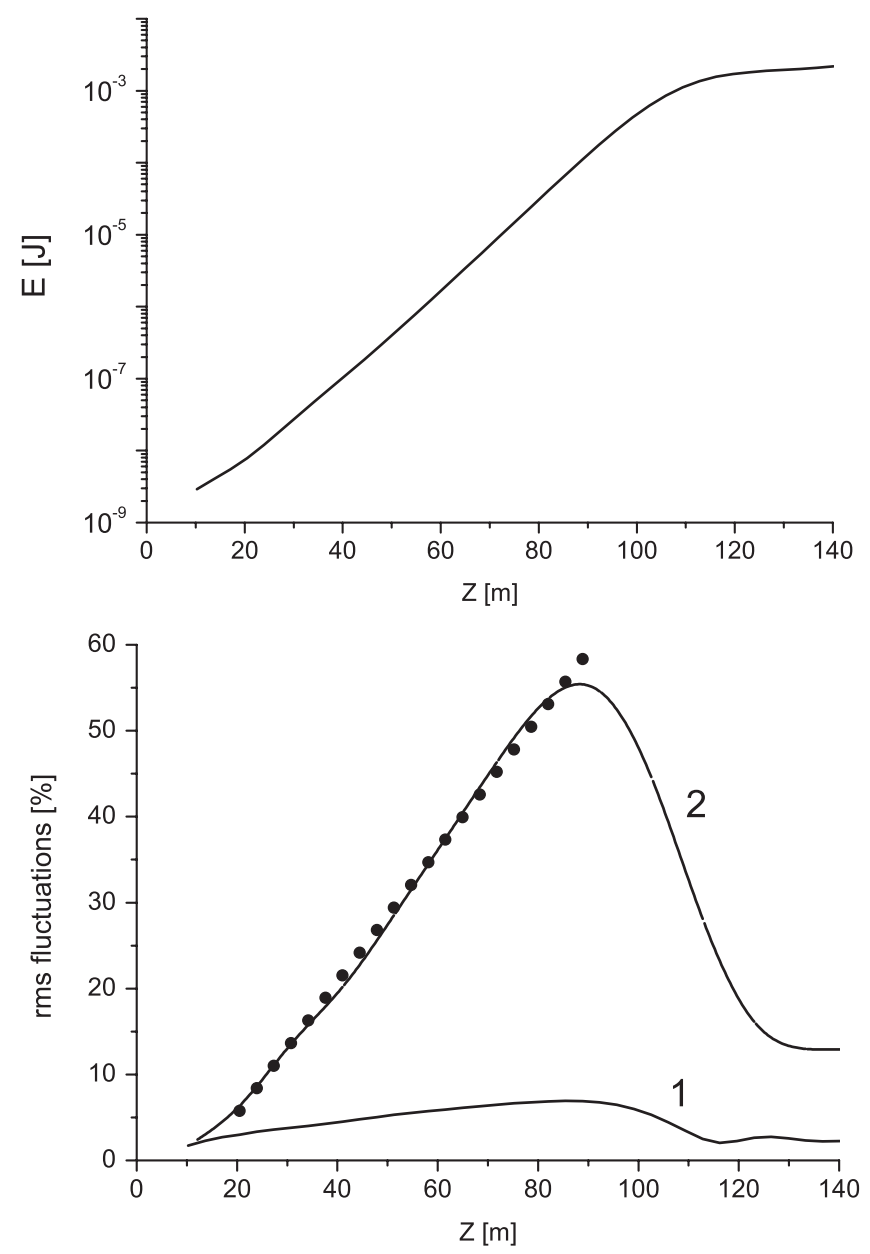

FIG. 1. Results of numerical simulations with FAST. Radiation pulse energy (upper plot) and relative rms fluctuations (lower plot) versus undulator length for SASE1 undulator, operating at $0.1 \mathrm{~nm}$ [4]. Curve 1: intrinsic SASE fluctuations (stable electron beam); curve 2: with $10 \%$ rms fluctuations of bunch compression factor. Dots are calculated according to formula (1) with $L_{g}=$ $13 \mathrm{~m}$.

of short intense bunches in a compression scheme was proposed in [11]. Our scheme is based on the exploitation of an optically modulated electron beam. The concept has some similarities with a current-enhanced SASE scheme [12], although there are essential differences.

\section{STABILIZATION SCHEME DESCRIPTION}

Our stabilization scheme operates in five steps (a sketch is shown in Fig. 2). First, we reduce the compression factor in the main compression system. Note that a positive side effect of this step is to relax tolerances by the same amount. Second, we modulate the electron beam in energy by interaction with a laser in a short undulator just after the last bunch compressor (BC). Third, we convert energy modulation into a relatively small density modulation in a dispersion section (small chicane). Fourth, the beam propagates through the accelerator, accumulating energy modulation due to longitudinal space charge (LSC). Note that this modulation is much larger than that induced by the laser. Fifth, somewhere in front of the x-ray undulator we insert another dispersive element (e.g. a chicane [13]) to get density spikes with the design current. The transformation of the longitudinal phase space is nonlinear here, so that the density modulation includes also harmonics of the laser wavelength. It is important that we overbunch the beam (see Fig. 3), i.e. that the energy modulation is larger than what is needed to get maximal current for a given uncorrelated energy spread and $R_{56}$ of the chicane.

This treatment has the effect of achieving a reduced sensitivity of the FEL output on rf jitters. In fact, when the compression factor in the main compression system $C$ increases (decreases) due to rf jitters, the energy modulation due LSC is stronger (weaker) than in the case of nominal compression. As a result, the beam is more strongly (or weakly) overbunched in the last chicane. As a consequence, the enhancement of the current is smaller (larger). It follows that the product of the current enhancement by $C$ remains nearly constant over a wide range of a compression factor change.

Note that LSC is the most simple and robust collective effect to be used in such stabilization. For a typical parameter range of linacs and compression systems of hard $\mathrm{x}$ ray FELs, the longitudinal space charge effect falls into a comfortable regime when $\sigma_{\perp} \ll \gamma \lambda \ll b$. Here $\sigma_{\perp}$ is a transverse size of the beam, $\gamma$ is relativistic factor, $\lambda=\lambda / 2 \pi$ is the reduced modulation wavelength, and $b$ is a transverse size of a vacuum chamber. In other words, one deals with "pencil beam" limit on the one hand, and

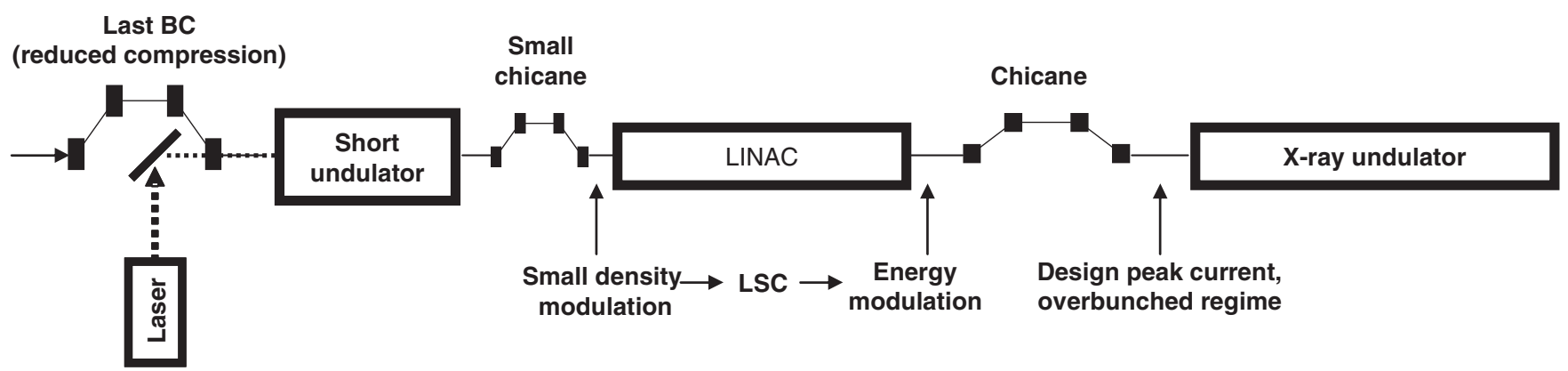

FIG. 2. Conceptual scheme for stabilization of SASE FEL operation. 


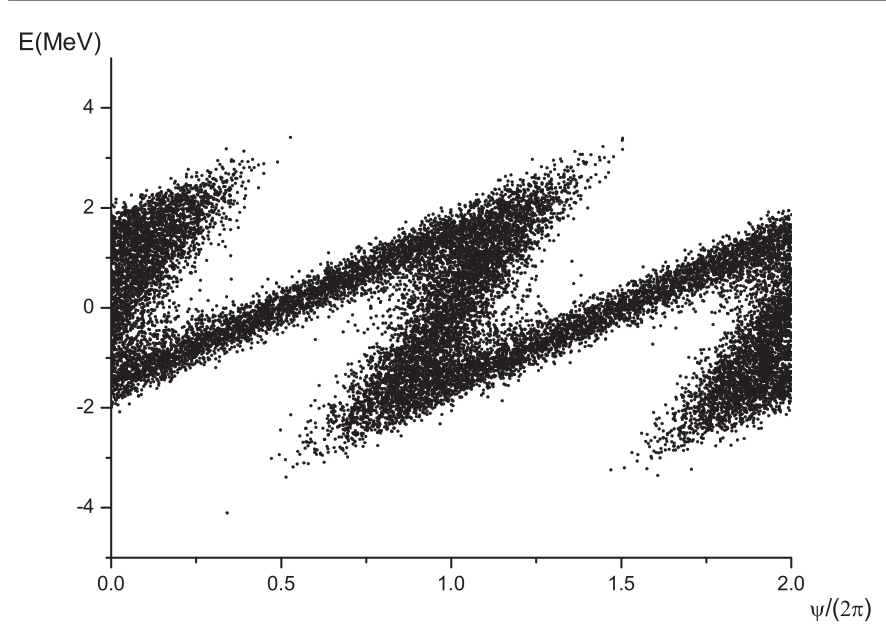

FIG. 3. A fraction of the longitudinal phase space after the last chicane of stabilization scheme.

with free-space limit on the other hand. Longitudinal space charge impedance scales as $\left(\lambda \gamma^{2}\right)^{-1} \ln \left(\lambda \gamma / \sigma_{\perp}\right)$, i.e. it depends on two well-defined parameters, wavelength and beam energy, and only weakly (logarithmically) depends on the transverse size. Therefore the effect can be easily calculated, and it is sufficiently strong for short wavelengths even at high beam energies.

\section{APPLICATION TO THE EUROPEAN XFEL}

We illustrate the operation of the stabilization scheme with a numerical example for the European XFEL (see Fig. 4). We consider the "standard" compression case [4], and we assume that the beam after the last bunch compressor (BC2) consists of a linearly compressed (by a factor 100) Gaussian bunch with peak current of $5 \mathrm{kA}$, rms length of $15 \mu \mathrm{m}$, and uncorrelated energy spread of $1 \mathrm{MeV}$.

We reduce the compression by factor 1.7 , thus getting $3 \mathrm{kA}, 25 \mu \mathrm{m}$, and $0.6 \mathrm{MeV}$ after BC2. We suppose that an optical replica synthesizer (ORS) [14] is installed after BC2. Subsequently, we modulate the beam in energy with an amplitude of $100 \mathrm{keV}$, in the first ORS undulator. To this purpose we use a Ti:S laser with a wavelength of $800 \mathrm{~nm}$ and a few MW peak power. When the beam passes the ORS chicane (with $R_{56}=150 \mu \mathrm{m}$ ), the energy modulation is converted into a $5.5 \%$ density modulation.

The radiator of the ORS is not used, and the beam is subsequently accelerated in the main linac from 2 to 17.5 GeV. Because of LSC, an energy modulation of about 1.6 MeV is accumulated at the nominal current of $3 \mathrm{kA}$. Calculations were performed as described in [15].

We propose to install another chicane (BC3) after the collimation system (see Fig. 4). Setting $R_{56} \simeq 3.3 \mathrm{~mm}$ we can obtain density spikes with a current of about $5.2 \mathrm{kA}$ and full width of about $430 \mathrm{~nm}$ in the overbunched regime (see Fig. 5).

By varying the compression factor in the main compression system and calculating the current distribution after BC3, one can see from Fig. 6 that the variations of peak current are only a few percent when the compression factor after $\mathrm{BC} 2$ changes by $\pm 30 \%$.

This shows that we can stabilize the current, which enters the argument of the exponential function in the expression for the radiation power. The FEL pulse energy, however, depends on the pulse width too. Consider the width of the envelope and that of a spike in Fig. 5-they both depend on the variation of the compression factor $C$ in the main compression system. For stronger compression, for example, the envelope shrinks (i.e. the number of highcurrent spikes, contributing to lasing, is smaller than in a nominal case), but the width of a spike gets larger, so that these two effects compensate each other to some extent. Although the width of the spike is visibly larger than the FEL coherence length (about $100 \mathrm{~nm}$ ), short-pulse effects on FEL operation cannot be completely neglected, especially in the case of low $C$. Moreover, both the energy chirps induced across the spikes by LSC in front of and in the x-ray undulator [16], have to be taken into account. Although contribution from these energy chirps is quite a small correction to the FEL gain in our numerical example, it is compression dependent and finally influences the SASE stability. Thus, to judge the overall stability, one needs to perform FEL simulations.

The FEL process with our modified beams has been calculated with the 1D version of the code FAST, taking into account the above-mentioned effects. For each value

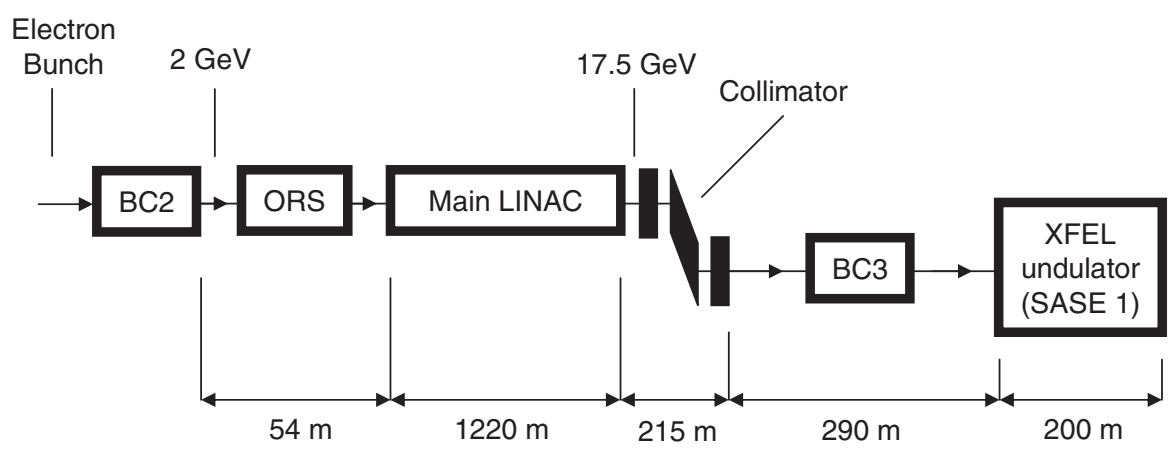

FIG. 4. Possible realization of the stabilization scheme at the European XFEL. 

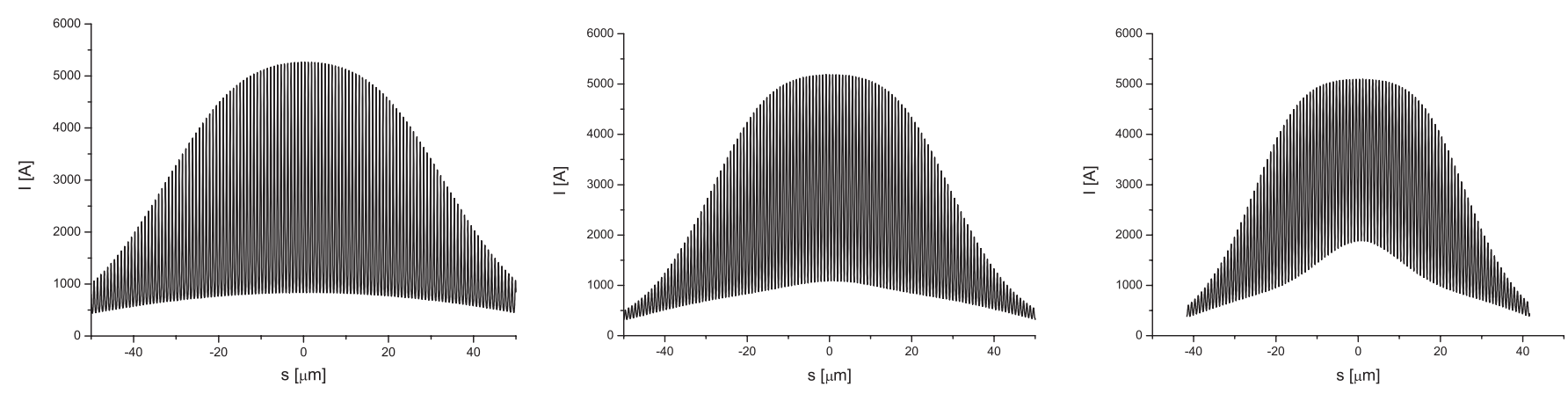

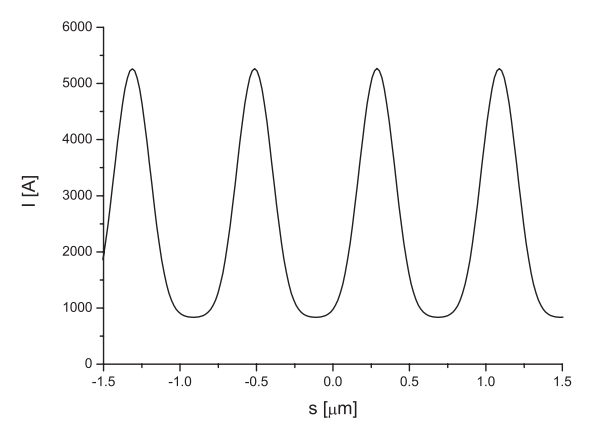

(a)

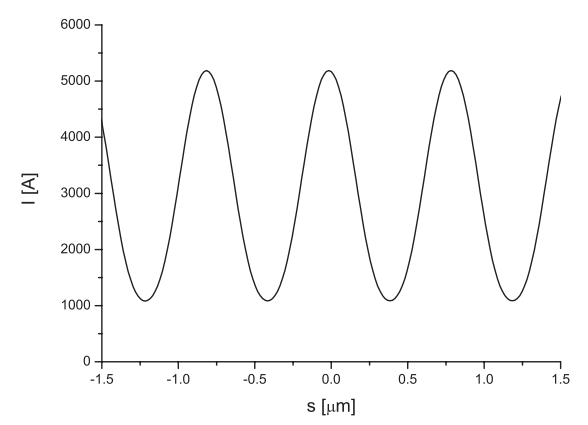

(b)

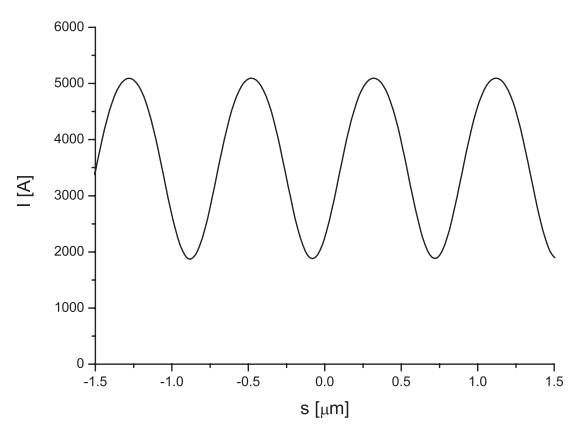

(c)

FIG. 5. The current distribution after BC3 (upper plots) and its enlarged fraction (lower plots) for $\Delta C / C_{0}=-20 \%$ (a), $\Delta C / C_{0}=0$ (b), and $\Delta C / C_{0}=20 \%$ (c).

$\Delta C / C_{0}$ in the main compression system we calculated the FEL pulse energy (averaged over an ensemble of shot noise realizations) for a standard compression case and for the case when the stabilization scheme is applied. The results for the end of the exponential gain regime (where the SASE fluctuations are the strongest) are presented in Fig. 7. For fair comparison, one should remember that, for the same variations of $\mathrm{rf}$ parameters, the variations of compression factor are smaller by a factor 1.7 when our scheme is used. Thus, for the same rf jitters one would

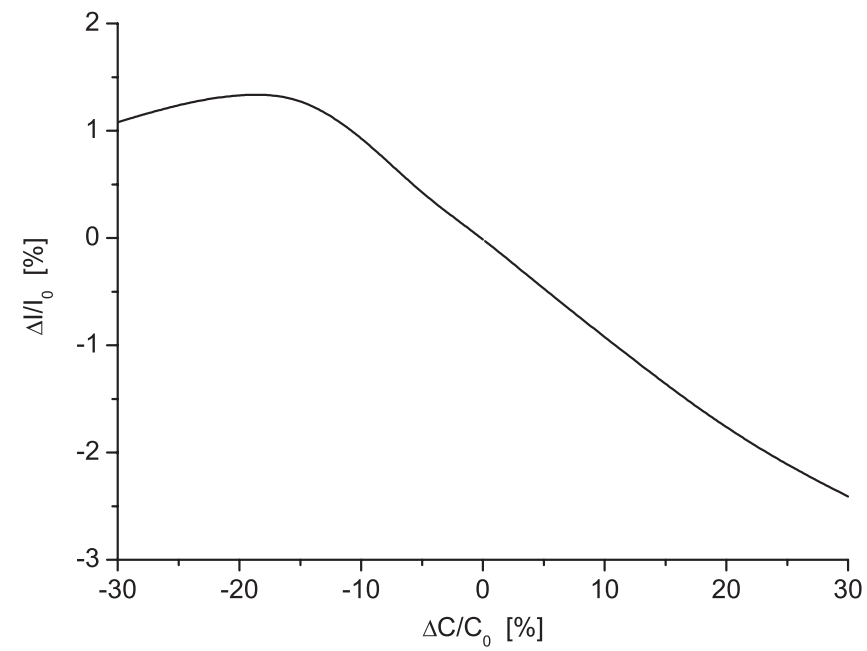

FIG. 6. Deviation of peak current after BC3 versus deviation of the compression factor in the main compression system (after $\mathrm{BC} 2)$. reduce SASE pulse-energy fluctuations (not including intrinsic fluctuations) by a factor 10 in all the parameter range in Fig. 7. Alternatively, one can compare rf jitter tolerances for the same SASE fluctuations. For given SASE fluctuations, the ratio of $\sigma_{C} / C_{0}$ for the two considered cases is 5-6 in Fig. 7, depending on the allowed $\sigma_{E} / E$. Thus, in the numerical example considered here,

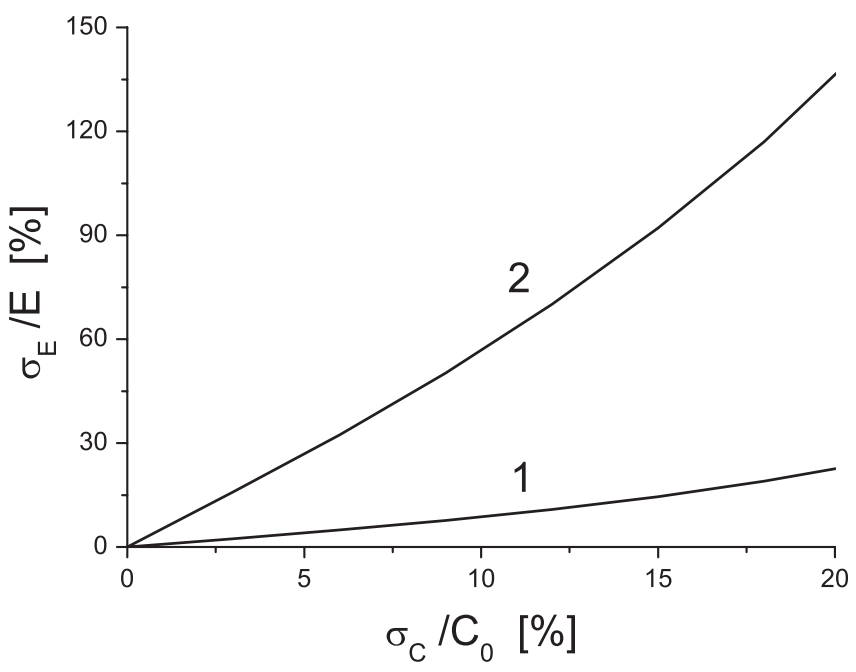

FIG. 7. Rms fluctuations of FEL pulse energy at the end of exponential gain regime $(z=90 \mathrm{~m})$ versus rms fluctuations of bunch compression factor for two cases. Curve 1: stabilization scheme is applied; curve 2: standard compression scheme. Intrinsic SASE fluctuations are excluded. 
the application of the stabilization scheme would allow one to loosen tolerances by a factor of $(5-6) \times 1.7 \simeq 8-10$ in all the parameter range in Fig. 7.

\section{DISCUSSION}

In this paper we did not consider enhancement of the current [12], keeping it at the XFEL design value of $5 \mathrm{kA}$ for fair comparison with the standard compression scheme. Note that such an enhancement is easily possible (for instance, just by increasing the compression factor in the main compression system back to its original value, and/or by changing parameters of the optically modulated beam and the chicane). An important feature of our scheme is that the energy modulation due to LSC is much larger (more than factor 10) than that induced by the laser. In other words, the laser power can be reduced by more than 2 orders of magnitude with respect to a case where no LSC is present.

Also note that we did not consider a specific design of the chicane. In fact, this might be influenced by coherent (CSR) and incoherent (ISR) synchrotron radiation. The length of the chicane will mainly be determined by ISR effects: for a chosen operating point and the considered $R_{56}$, it cannot be shorter than $15-30 \mathrm{~m}$ (but can be reduced for a different operating point, for instance with larger energy spread and modulation). CSR effects on longitudinal and transverse dynamics are greatly reduced due to the $R_{51}$ effect, so that, according to our estimates, decrease of the final peak current and increase of emittance are small corrections. Subsequent reduction of the FEL gain can be compensated by an increase of the current as described above.

A possible challenge for the XFEL beam formation system is the LSC driven microbunching instability [17]. A laser heater [17] is supposed to suppress such an instability and it is included in the European XFEL design. It must be remarked that introducing one more chicane, as in our scheme, would increase the microbunching instability gain, so that larger energy spread might have to be generated in the heater. In this case, the $R_{56}$ of the $\mathrm{BC} 3$ should be reduced proportionally, and the density modulation generated in the ORS should be increased by the same factor. At the considered operation point, the energy spread was smaller than that generated in the undulator due to quantum diffusion and gave very weak correction to the FEL gain. With an increased energy spread the FEL gain reduction can be compensated by a moderate increase of beam current.
Finally, note that the realization of the proposed scheme would automatically allow one to use a method for timing an XFEL source to high-power lasers [15]. Since the amplitude of the density modulation necessary in the stabilization scheme is an order of magnitude larger than that used in [15], the power of visible radiation produced after an $\mathrm{x}$-ray undulator would increase by 2 orders of magnitude.

[1] A. M. Kondratenko and E. L. Saldin, Part. Accel. 10, 207 (1980).

[2] Ya. S. Derbenev, A.M. Kondratenko, and E.L. Saldin, Nucl. Instrum. Methods Phys. Res. 193, 415 (1982).

[3] J. B. Murphy and C. Pellegrini, Nucl. Instrum. Methods Phys. Res., Sect. A 237, 159 (1985).

[4] M. Altarelli et al., Technical Design Report, DESY Report No. DESY 2006-097, Hamburg, 2006 (see also http:// xfel.desy.de).

[5] J. Arthur et al., Conceptual Design Report, Report No. SLAC- R593, Stanford, 2002 (see also http://wwwssrl.slac.stanford.edu/lcls/cdr).

[6] SCSS X-FEL: Conceptual design report, RIKEN, Japan, May 2005. (see also http://www-xfel.spring8.or.jp).

[7] E. L. Saldin, E. A. Schneidmiller, and M. V. Yurkov, The Physics of Free Electron Lasers (Springer, Berlin, 1999).

[8] E. L. Saldin, E. A. Schneidmiller, and M. V. Yurkov, Nucl. Instrum. Methods Phys. Res., Sect. A 429, 233 (1999).

[9] E. L. Saldin, E. A. Schneidmiller, and M. V. Yurkov, Opt. Commun. 235, 415 (2004).

[10] M. Dohlus and T. Limberg, in Proceedings of the 27th International Free Electron Laser Conference (FEL 2005), Stanford, 2005, p. 250.

[11] H. Schlarb and R. Brinkmann, in Proceedings of the Particle Accelerator Conference, Vancouver, BC, Canada, 1997 (IEEE, New York, 1997), p. 1572.

[12] A. Zholents et al., in Proceedings of the 26th International Free Electron Laser Conference (FEL 2004), p. 582.

[13] Note that the sign of $R_{56}$ does not really matter so that a dogleg or other configurations can also be used.

[14] E. L. Saldin, E. A. Schneidmiller, and M. V. Yurkov, Nucl. Instrum. Methods Phys. Res., Sect. A 539, 499 (2005).

[15] G. A. Geloni, E. L. Saldin, E. A. Schneidmiller, and M. V. Yurkov, Opt. Commun. 281, 3762 (2008).

[16] G. A. Geloni, E. L. Saldin, E. A. Schneidmiller, and M. V. Yurkov, Nucl. Instrum. Methods Phys. Res., Sect. A 583, 228 (2007).

[17] E. L. Saldin, E. A. Schneidmiller, and M. V. Yurkov, Nucl. Instrum. Methods Phys. Res., Sect. A 528, 355 (2004). 